\title{
Facile Assembly of Cadmium Sulfide Quantum Dots on Titanate Nanobelts for Enhanced Nonlinear Optical Properties
}

\author{
Miao Feng, ${ }^{\dagger}$ Hongbing Zhan, ${ }^{*}{ }^{\dagger}$ and Lei Miao* ${ }^{*}$ \\ College of Materials Science and Engineering, Fuzhou University, 2 Xueyuan Road, Fuzhou 350108, People's Republic of \\ China, and Key Laboratory of Renewable Energy and Gas Hydrate, Guangzhou Institute of Energy Conversion, Chinese \\ Academy of Sciences, Nengyuan Road, Wushan, Tianhe, Guangzhou, 510640, People’s Republic of China
}

\begin{abstract}
A facile route to assemble cadmium sulfide (CdS) quantum dots (QDs) uniformly on the surface of titanate nanobelts (TNBs) through electrostatic interactions is demonstrated. The photophysical properties of the resulting TNB-CdS nanostructured composite, including optical limiting properties, were studied using ultraviolet-visible absorption spectroscopy, photoluminescence spectroscopy, and the open aperture Z-scan technique in the nanosecond regime using a laser with a wavelength of $532 \mathrm{~nm}$. The linear and nonlinear optical properties of this composite nanostructure were strongly influenced by a possible charge/energy transfer process between the QDs and TNBs. The as-prepared TNB-CdS composite offers an optical limiting effect that is superior to that of unmodified CdS QDs and TNBs. The main contributors to the enhanced optical limiting effect in the TNB-CdS composite were a combination of nonlinear scattering and increased nonlinear absorption resulting from efficient charge/energy transfer at the CdS/ TNB interface.
\end{abstract}

KEYWORDS: composite nanostructure $\bullet$ Z-scan $\bullet$ optical limiting $\bullet$ nanosecond laser

\section{INTRODUCTION}

1 itanate materials have attracted much attention because of their large surface area, uniform nanochannels, low toxicity, and excellent optical and ionexchange properties (1). The biggest advantage of titanate materials is that their chemical and physical properties can be tailored by adjusting their nanostructures to satisfy the specific needs of new applications. Recent achievement of large-scale and cost-effective synthesis of titanate materials by several methods (including a template method (2), a surfactant-assisted self-assembling process (3), and a strongly alkaline hydrothermal treatment (4)) pave the way for the wide use of titanate-based pigments, gas sensors, photocatalysts, and photoelectronic devices.

Multilayered titanates with exchangeable alkali metal ions located in the interlayer region are a sort of layered transition metal oxide. These act as photocatalysts and are very useful as hosts for some guest species, such as phthalocyanine (5), pseudoisocyanine dye (6), rare earth ions (7), and Ru(bpy) ${ }_{3}{ }^{2+}$ (8). From a purely structural perspective, one-dimensional titanate nanomaterials (1D TNMs, including titanate nanotubes, nanowires, nanobelts, and nanorods) with nanoscale inner cavities and exposed outer surfaces possess high specific surface areas, making them unique reaction vessels for building nanoscale photoactive components. It is there-

\footnotetext{
* Corresponding author. Tel: +86-591-22866512. E-mail: hbzhan@fzu.edu.cn (H.Z.); miaolei@ms.giec.ac.cn (L.M.).

Received for review January 3, 2010 and accepted March 11, 2010

${ }^{\dagger}$ Fuzhou University.

* Chinese Academy of Sciences.

DOI: $10.1021 /$ am $100003 p$

2010 American Chemical Society
}

fore of great interest to encapsulate or adorn 1D TNMs with metal sulfide (CdS (9)), metal oxide (CuO (10)), and noble metal nanoparticles (NPS) (Ag and Au (11)). Metal NPS present in this architecture exhibit high reactivity and enhanced catalytic efficiency, surface plasmon absorption, and nonlinear optical (NLO) properties compared with their unmodified equivalents, giving them potential for optoelectronic, photocatalytic, and photovoltaic applications.

However, there still is a huge contrast between the potential and reality of using 1D TNMs in diverse practical applications on a widespread scale. Although pristine TNMs have hydrophilic surfaces with functional hydroxyl groups during the preparation process (1b), strong van der Waals interactions result in aggregation, preventing facile individualization. Unlike carbon nanotubes (CNTs), for $1 \mathrm{D}$ TNMs, the chemical inertness of the titanate surface usually leads to difficulties in achieving reliable surface modification by chemical treatments. From a materials chemistry perspective, only a small amount of research has concentrated on the surface modification and functionalization of 1D TNMs (12), possibly because of the poor solubility of titanate in water and organic solvents.

In recent years, the search for potential optical limiters to protect human eyes and sensors from the threat of intense laser pulses has motivated many researchers to explore new materials which exhibit superior optical limiting (OL) properties. Generally, the OL materials should strongly attenuates high intensity light and potentially damaging light such as focused laser beams to protect human eyes and sensors, while allowing for the high transmission of ambient light so as not to degrade normal vision (13). Up to now, strong OL 
Scheme 1. Synthetic Pathway to the TNB-CdS Composite Nanostructures

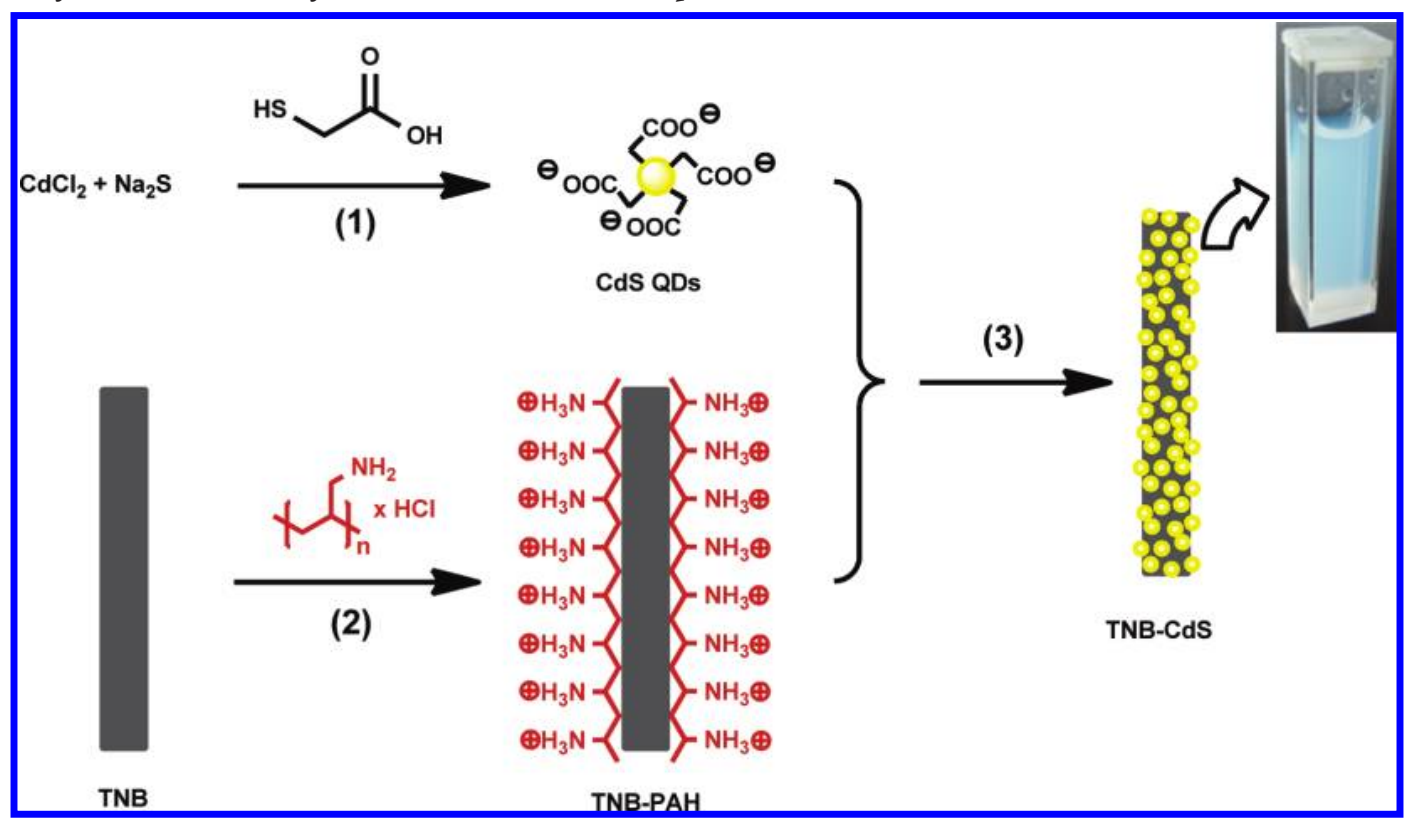

properties have been intensively studied in a wide range of materials, including organic materials (14), carbon black and CNTs (15), fullerenes (16), graphene (17), and metal and semiconductor NPs (18) by exploiting passive OL mechanisms, particularly nonlinear absorption (NLA), nonlinear scattering (NLS), and nonlinear refraction (NLR). Despite the improvement in OL properties because of better design strategy, no single OL material or mechanism meets all of the requirements for an ideal optical limiter. CNTs exhibit unique broadband OL properties from the visible to the near infrared in the nanosecond regime, which arise from NLS because of the formation of scattering centers consisting of solvent bubbles and ionized carbon microplasmas (15b). Nevertheless, the weaknesses of CNTs as OL materials, such as the pulse duration dependence, high limiting threshold, and low linear transmission, hamper their practical use. Because of the extensive $\pi$ electron conjugation, fullerene shows NLA properties in a solid host, whereas in solution the NLA is supplemented by NLS and NLR (13d, 16). However, the low solubility of fullerene in a plethora of solvents, especially in water, is the major limiting factor on further studies and applications for this pioneering material, because the exclusive fullerene's properties are strictly related to the isolation of individual molecules. Research indicates that maximum OL performance can be expected to occur when the multiple nonlinear mechanisms are used in concert (13d). Many efforts have been invested into the research of the combinations of NLO materials in cascaded geometries (19) in an attempt to allow for the fabrication of composite OL materials to fully fulfill the requirements in a real application, such as large NLO response, good optical quality, mechanical stability, easy preparation procedures, and low cost. In the past years, some optical nonlinear media with NLA properties (e.g., two photon absorption (TPA) or reverse saturable absorption (RSA)) have been covalently linked to CNTs, resulting in significant improved OL performances mainly contributed by electron donor to acceptor interactions (20). Also, polycrystalline metals and semiconductor quantum dots (QDS) coated CNTs presented stronger OL effect compared to pure CNTs at $532 \mathrm{~nm}$ with nanosecond laser pulses (21). The dominant mechanism will depend on the material composition and device configuration.

However, reports on OL effects of decorated 1D TNMs with metal and semiconductor QDs are few as yet. Semiconductor QDs with large NLA have shown great potential as NLO materials, especially for ultrashort pico-femtosecond laser pulses. The generally accepted underlying OL mechanisms for QDs are TPA and/or multiphoton absorption (MPA) for pico-femtosecond laser pulses and free-carrier absorption (FCA) and/or NLS for nanosecond laser pulses. Our previous results revealed that the existent energy/charge transfer from the excited CdS NPs to the CNTs or graphene can result in a large OL effect $(21 \mathrm{c}, 22)$. Recently, we observed NLO properties in titanate nanobelts (TNBs) at $532 \mathrm{~nm}$ with nanosecond laser pulses, therefore, it is expected that combining the 1D TNBs with optoelectronically active QDs would lead to some interesting and significative consequences, including OL enhancement. From the perspective of exploring new OL materials, we present here a facile method to assembly of cadmium sulfide QDs on 1D TNBs, and the enhanced NLO properties of the resultant combination compared to those of individual components at $532 \mathrm{~nm}$.

To increase the solubility of TNBs in water and produce electric charge on their surface, the TNBs were pre-functionalized with water-soluble poly(allylamine hydrochloride) (PAH). PAH is a commercial cationic polyelectrolyte, which can be used to impart a surface charge to neutral NPs, enabling them to be dispersed in aqueous solution (23). Uniform decoration of carboxyl-anchored CdS QDs on the TNBs was achieved through electrostatic interactions, as illustrated in Scheme 1, which can be extended to fabricate a series of novel titanate-nanoparticle composite nanostructures with tailored electronic or optical properties. Large variation in the shape and size of QDs hinders their size- 
dependent optical and electrical properties, lowering the performance of their optoelectronic devices. The greatest advantage of the method used herein is the realization of homogeneous QDs with narrow size distributions at high loadings on the surface of the TNBs. The as-prepared TNBCdS composites offer an OL effect that is superior to that of CdS QDs and TNBs alone. The enhanced OL effect mainly arises from the combination of NLS and enhanced NLA which result from efficient charge/energy transfer at the CdS/ TNB interface.

\section{EXPERIMENTAL SECTION}

Materials. All Chemicals were purchased from Aldrich and used as received. Deionized water was used in all of the aqueous solutions and washings. TNBs were synthesized by hydrothermal reaction of $\mathrm{TiO}_{2}$ powder in strongly alkaline $(\mathrm{NaOH})$ aqueous solutions (24).

Synthesis of Carboxyl-Anchored CdS QDs. On the basis of a typical procedure for the synthesis of carboxyl-anchored CdS QDs (25), we added thioglycolic acid (TGA, $2.15 \mathrm{mmol}, 150 \mu \mathrm{L}$ ) to an aqueous solution of cadmium chloride $\left(\mathrm{CdCl}_{2} \cdot 2.5 \mathrm{H}_{2} \mathrm{O}\right.$, $0.3 \mathrm{mmol}, 270 \mathrm{~mL}$ ) and stirred for $15 \mathrm{~min}$, forming a turbid blue solution. The $\mathrm{pH}$ of the solution was adjusted to 11 with aqueous sodium hydroxide $(0.01 \mathrm{~mol} / \mathrm{L})$. Aqueous sodium sulfide $\left(\mathrm{Na}_{2} \mathrm{~S} \cdot 9 \mathrm{H}_{2} \mathrm{O}, 0.15 \mathrm{mmol}, 10 \mathrm{~mL}\right)$ was added dropwise and the solution was then stirred for $1 \mathrm{~h}$ at room temperature in the dark. The yellow reaction mixture was slowly concentrated by distillation under reduced pressure, followed by precipitation using anhydrous methanol to yield the watersoluble, carboxyl-anchored CdS QDs.

Surface Functionalization of TNBs. The surface of the TNBs was functionalized by suspending TNBs $(50 \mathrm{mg})$ in an aqueous solution $(500 \mathrm{~mL})$ containing sodium chloride $(0.5 \mathrm{~mol} / \mathrm{L})$ and PAH $\left(M_{\mathrm{w}} \approx 56,000,5 \mathrm{~g} / \mathrm{L}\right)$. The suspension was sonicated for 30 minutes in a high-power sonic bath, and then gently agitated over night to ensure complete and uniform dispersal of the TNBs. The white precipitate (TNB-PAH) collected by centrifugation of the suspension was washed several times with deionized water and ethanol.

Assembly of CdS QDs on TNBs. An aqueous solution of TNB$\mathrm{PAH}(0.5 \mathrm{~g} / \mathrm{L}, 20 \mathrm{~mL})$ was added to the suspension containing the CdS QDs $(30 \mathrm{~mL})$ using a magnetic stirrer. The reaction mixture was stirred in the dark for $24 \mathrm{~h}$ at room temperature. Excess QDs were removed by centrifugation at $8000 \mathrm{rpm}$ followed by rinsing several times with deionized water to give high-quality CdS QD-functionalized TNBS

Characterization. Transmission electron micrographs (TEM) of the samples were obtained on a JEOL JEM-2010 microscope. The samples were dispersed in water, and then a drop of the solution was placed on a copper grid and dried before it was transferred into the TEM sample chamber. X-ray diffraction (XRD) data were collected using a Philips XPert-MPD X-ray diffractometer with $\mathrm{Cu}-\mathrm{K} \alpha$ radiation. Raman spectra were recorded on a Renishaw 2000 Raman spectrometer (514.5 nm). Ultraviolet-visible (UV-vis) absorption spectra were obtained on a Shimadzu UV-2450 spectrophotometer. Photoluminescence (PL) spectra were measured on a Edinburgh FL/FS TCSPC 920 spectrofluorophotometer. All experiments were performed at room temperature.

Nonlinear Optical Measurements. All the nonlinear optical (NLO) experiments described were performed using 8 ns Gaussian pulses from a Q-switched Nd:YAG laser. The beam was spatially filtered to remove the higher order modes and focused with a lens at a focal length of $30 \mathrm{~cm}$. The laser was operated at $532 \mathrm{~nm}$ with a pulse repetition rate of $1 \mathrm{~Hz}$. The scattered light was collected at $45^{\circ}$ to the $z$-axis. The nanostructures were dispersed in water and contained in quartz cells with a path
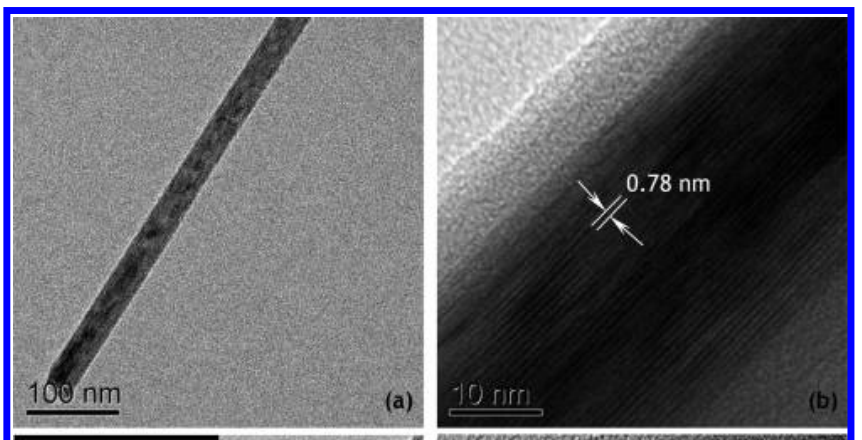

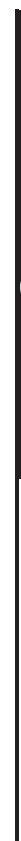

(c)

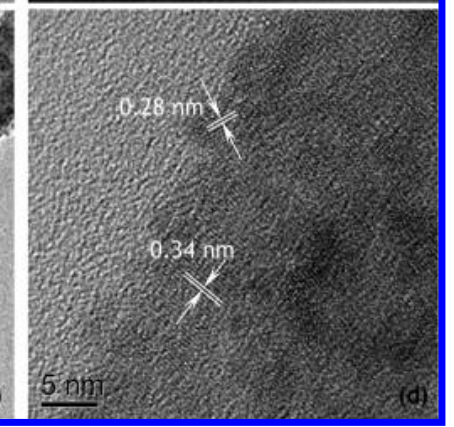

FIGURE 1. TEM and HRTEM images of the $(a, b)$ TNB-PAH and $(c, d)$ TNB-CdS samples. The inset in $\mathrm{c}$ is the SAED pattern taken from the area designated by the rectangle.

length of $5 \mathrm{~mm}$. The linear transmittance of the tested sample was adjusted to $70 \%$. The OL curves, which can be plotted as normalized transmission versus input fluence, were extracted from the open aperture Z-scan data for the samples.

\section{RESULTS AND DISCUSSION}

Figure $1 \mathrm{a}-\mathrm{d}$ shows representative TEM images of TNB-PAH (Figure 1a,b) and TNB-CdS (Figure 1c,d). The TNB-PAH image showed TNBs with a smooth surface and an average diameter of $\sim 35 \mathrm{~nm}$. The TNBs were individually dispersed in water, indicating that the van der Waals interaction usually observed between unmodified TNBs has been effectively overcome by the electrostatic repulsion created between the nanobelts by functionalization with PAH. The layered nature of the titanate structures was observed clearly in the high-resolution TEM (HRTEM) image of TNB-PAH (Figure $1 \mathrm{~b}$ ). The interlayer spacing along the belt was found to be about $0.78 \mathrm{~nm}$, which is consistent with the $d_{020}$ of an orthorhombic protonic lepidocrocite structure (26). PAH formed a homogeneous amorphous polymer layer with a thickness of about $8 \mathrm{~nm}$ that fully covered the surface of the TNB. Figure 1c,d confirms the successful formation of the TNB-CdS assembly with CdS QDs bound to the surface of the nanobelts. CdS QDs with uniform size and shape dispersed homogeneously and strongly adhered to the TNB surface. A selected area electron diffraction (SAED) pattern taken from the region of the nanostructure shown in a rectangle (inset of Figure 1c) is consistent with the (200) and (110) diffraction planes of the orthorhombic lepidocrocite structure of TNBs, as well as the lattice parameters of cubic $\mathrm{CdS}$. The diffraction rings of CdS were not legible, indicating a thin and even coating of CdS QDs along the entire length of the TNBs. Figure $1 \mathrm{~d}$ is a HRTEM image of the TNB-CdS assembly, which reveals the trend of the QDs to associate with the surface of the nanobelt. Single crystalline cubic CdS 


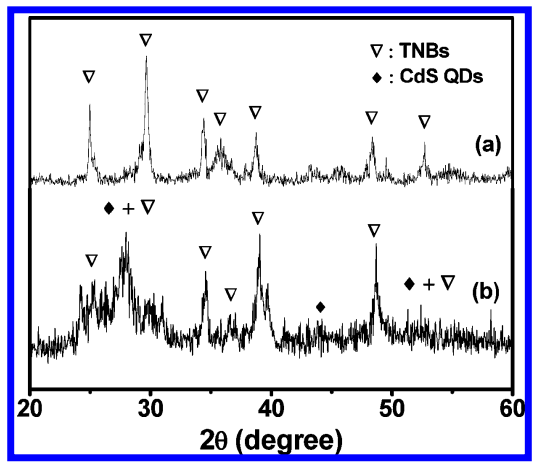

FIGURE 2. XRD patterns of the (a) TNB and (b) TNB-CdS samples.

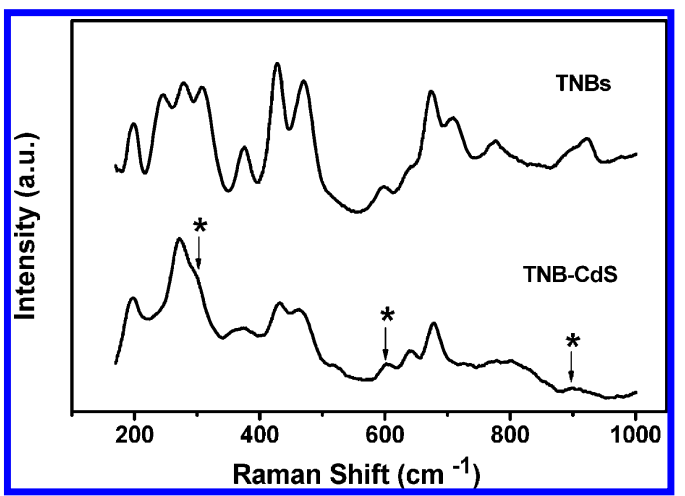

FIGURE 3. Raman spectra of the TNB and TNB-CdS samples.

nanocrystals with a clearly resolved (111) lattice fringe spacing $\left(d_{111}=0.28 \mathrm{~nm}\right)$ were observed. Most of the CdS QDs have dimensions of 3-4 $\mathrm{nm}$. The interlayer distance measured perpendicular to the belt is $0.34 \mathrm{~nm}$, which could be assigned to the $d_{110}$ of an orthorhombic protonic lepidocrocite structure (26).

XRD patterns of the TNB and TNB-CdS samples are shown in Figure 2. The pristine TNBs exhibit peaks at $2 \theta=$ $24.9,29.6,34.4,35.8,38.8,48.3$, and $52.7^{\circ}$, which can be attributed respectively to the (110), (310), (312), (602), (113), (020), and (022) reflections of a titanate material with an orthorhombic protonic lepidocrocite structure (27). The TNBCdS sample shows an additional peak at $2 \theta=26.3^{\circ}$ consistent with the (111) reflection of CdS, confirming that CdS QDs are present on the surface of the TNBs. Two other CdS reflection peaks at $2 \theta=43.8$ and $51.7^{\circ}$ (which index to the CdS (220) and (311) planes, respectively) were absent, even though they are usually present in the XRD patterns of CdS materials. This is attributed to the small size of the crystallites and the capping TGA ligand on the CdS QDs (21c).

Figure 3 shows the Raman spectra obtained for the samples. For the TNBs, the bands observed in the spectrum agree fairly well with the reported Raman data for titanate materials (28). The differences in the relative intensities of the peaks may be attributed to the textured microstructure of the sodium trititanate nanobelts, which are mostly continuous and roughly parallel to each other. In the Raman spectrum of TNB-CdS, the attachment of CdS QDs caused the characteristic signals of the TNBs become weaker and broader. In addition, three small peaks are observed at around 300,600 , and $900 \mathrm{~cm}^{-1}$ that are assigned to the first and second longitudinal optical modes, and the third har-

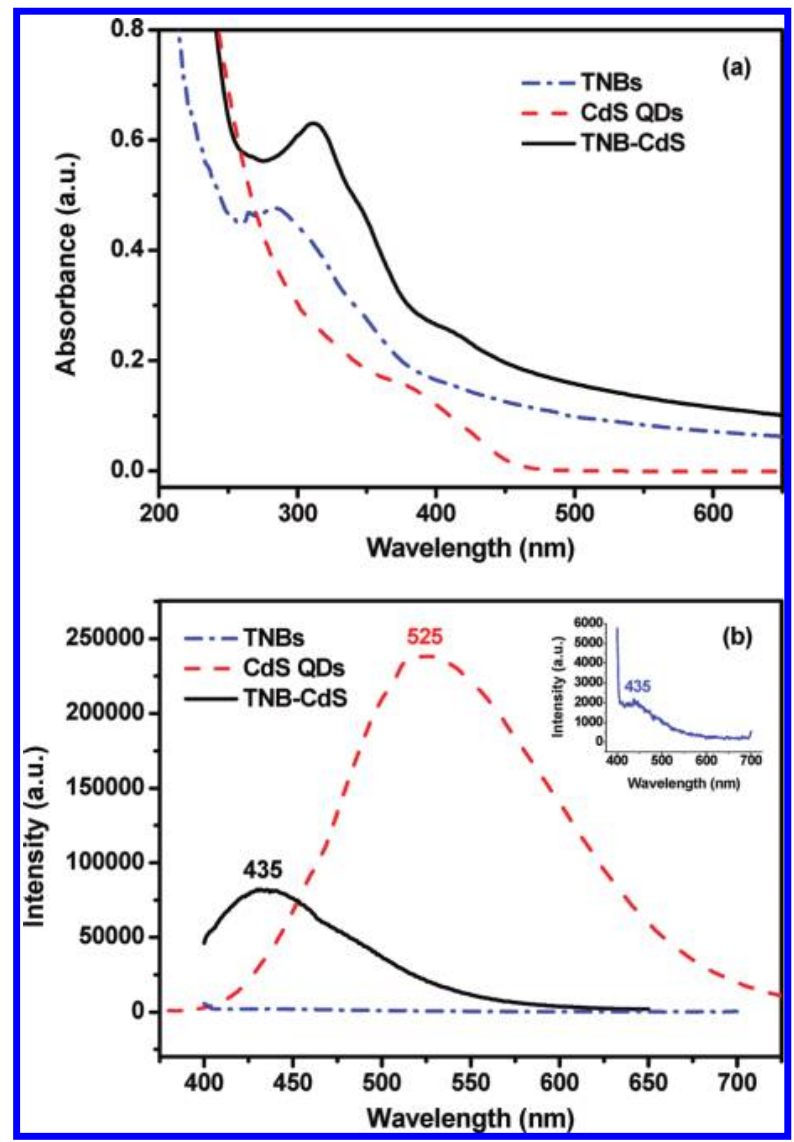

FIGURE 4. (a) UV-vis and (b) PL spectra ( $\lambda_{\mathrm{ex}}=375 \mathrm{~nm}$ ) of TNB, CdS $\mathrm{QD}$, and TNB-CdS samples suspended in aqueous solution at room temperature.

monic of the CdS QDs, respectively. The CdS QD signals are weak because the titanate phase is most abundant component in the composite nanostructure. The Raman spectrum is predominantly similar to that of titanate (26).

The linear optical properties of the TNB-CdS composite nanostructure were studied by UV-vis and PL spectroscopy. As shown in Figure 4a, the TNBs displayed a broad absorption with a maximum at around $280 \mathrm{~nm}$. It is known that $\mathrm{TiO}_{2}$ possesses a highly ionic lattice, the valence band of the lattice is composed of oxygen $2 p$ orbitals $\left(\mathrm{O}^{2-} 2 \mathrm{p}\right)$ strongly hybridized with Ti $3 \mathrm{~d}$ states, and the conduction band consists mostly of excited states of $\mathrm{Ti}^{4+}\left(\mathrm{Ti}^{4+} 3 \mathrm{~d}\right.$ ) (29). Therefore, the absorption peak is the result of the electronic transition from the $\mathrm{O}^{2-}$ antibonding orbital to the lowest unoccupied orbital of $\mathrm{Ti}^{4+}$ (26). CdS QDs typically show a band at around $380 \mathrm{~nm}$ in their UV-vis spectra, which corresponds to the first optically allowed transition between the electron state in the conduction band and the hole state in the valence band (30). The band gap energies estimated from the absorption spectra are 3.00 and $2.62 \mathrm{eV}$ for the TNBs and CdS QDs, respectively. After the assembly of CdS QDs on the TNBs, an obvious red shift of the peak maxima (ca. $26 \mathrm{~nm}$ ) was observed for the TNB absorption, whereas the typical absorption of the CdS QDs became even weaker and broader, with the active range being extended from 388 to $474 \mathrm{~nm}$ as a result of an interparticle coupling effect (31). The absorption spectrum of TNB-CdS did not show any new absorption features, indicating that charge diffusion or 
electronic interaction did not occur between the nanobelts and QDs in their ground state. The increased light absorption by the TNB-CdS composite compared to TNBs and CdS QDs alone can be explained by efficient charge separation at the junction between the materials. This is because a staggered (type II) band alignment exists between the two materials in the composite (32). The change in the position of the absorption bands is possibly due to the larger average size of the TNB-CdS composite compared to the unmodified TNBs.

The PL data in Figure $4 \mathrm{~b}$ shows that the TNBs emit at around $435 \mathrm{~nm}$, which results from shallow trap emission associated with oxygen vacancy (29b, 30). Upon excitation at $375 \mathrm{~nm}$, the CdS QDs exhibit one broad and strong emission band at around $525 \mathrm{~nm}$ which is caused by electron-hole recombination at surface traps (33). It is clear that the characteristic emission of CdS QDs between 400 and $700 \mathrm{~nm}$ is significantly reduced after attachment to the TNBs, whereas emission from the TNBs at $435 \mathrm{~nm}$ is enhanced considerably. This behavior shows that efficient charge/energy transfer occurs at the CdS/TNB interface. As shown in Scheme 1, the aqueous solution containing TNBCdS was blue in daylight. This suggests that interactions between the TNBs and QDs involve charge transfer of photoexcited electrons from the conductive band of the donor QDs to empty electronic states of the acceptor TNBs, resulting in nonradiative decay of the QD excited state (34). The efficient energy transfer from the QDs to the TNBs highlights the potential for TNB-CdS to behave as an effective light conversion material for photovoltaic applications (35).

Open aperture Z-scan experiments were carried out in the nanosecond regime at $532 \mathrm{~nm}$ to investigate NLO properties of the samples, and the results are shown in Figure 5. All of the Z-scan curves display a decrease in transmittance at the focal point of the laser where the input fluence is at its maximum, typical of a positive NLO effect induced by incident light. The minimum transmittances at $Z=0$ were 77,67 , and $65 \%$ for the TNBs, CdS QDs, and TNB-CdS, respectively. The transmittance curves of the CdS QDs and TNB-CdS are quite similar to each other, and their NLO performances are obviously better than that of the TNBs. However, TNB-CdS has the largest decrease in transmittance of the three samples, indicating that $\mathrm{OL}$ enhancement is achieved by decorating the TNBs with CdS QDs.

Figure $5 \mathrm{~b}$ depicts the open aperture Z-scan curves of the TNB-CdS sample at different input fluences. Using the Crank-Nicolson finite-difference scheme, the value for the nonlinear extinction coefficient $\beta$ was found unambiguously by fitting the $T(z)$ equation to the experimental data in Figure 5 (36):

$$
T(z)=\left[1 / \pi^{1 / 2}\right] \int_{-\infty}^{+\infty} \ln \left[1+q(z) \exp \left(-\xi^{2}\right)\right] d \xi
$$

where

$$
q(z)=\beta I_{0} L /\left[1+\left(z / Z_{0}\right)^{2}\right]
$$

$L$ is the sample thickness, $Z_{0}$ is the Rayleigh length, $Z$ is the sample position, and $I_{0}$ is the peak irradiation intensity at focus. $\zeta$ is the pulse width. The values of $\beta$ for TNBs, CdS QDs and TNB-CdS were calculated to be $1.21,1.73$, and $1.84\left(\times 10^{-14} \mathrm{~cm} / \mathrm{GW}\right)$ at $532 \mathrm{~nm}$, respectively. Comparing the values of $\beta$ between the three samples, it can be found that TNB-CdS has the largest nonlinear extinction coefficient, which verified the advantage of the composite system.

After referring to previous publications on the excellent OL properties of fullerene, CNTs, graphene, and their functionalized materials (37), this increased OL effect arises from the combination of NLS with NLA, as well as the efficient energy and/or electron transfer upon photoexcitation from electron donor CdS QDs to acceptor TNBs. For CdS particles in the case of the composite film, saturable absorption occurs at low intensity, and such saturation is mostly caused by one-phone bleaching (38). In general, the smaller particle size is, the larger blue shift of the absorption/emission band is. As a result, it makes the greater degree of overlap with the absorption peak of TNBs. This would help to promote charge transfer from CdS to TNBs. From the perspective of nonlinear optics, the smaller NPs have higher NLA because of quantum confinement effects, extracting more charge/ energy from incident laser beam and passing more along to TNBs than their bigger counterpart in the system. It is

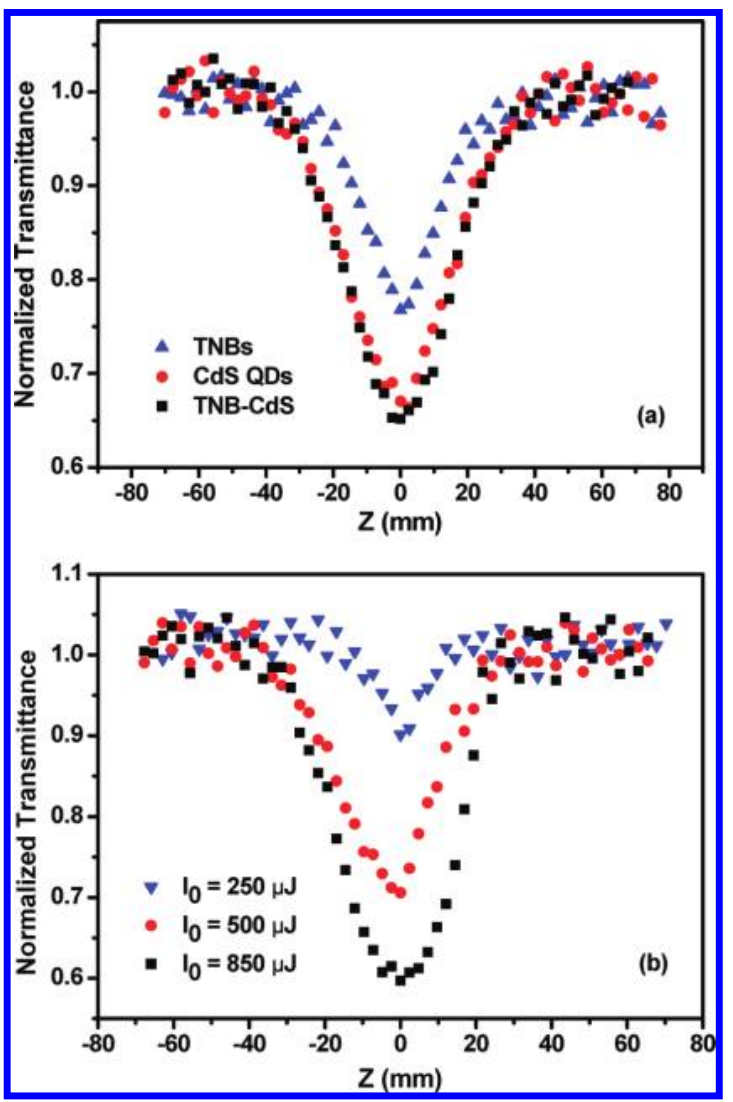

FIGURE 5. Open aperture Z-scan curves for $8 \mathrm{~ns}$ pulsed laser at 532 nm. (a) Z-scan of TNB, CdS QD, and TNB-CdS samples in aqueous solution with a linear transmittance of $70 \%$, and (b) Z-scan curves of TNB-CdS with different input fluence. 


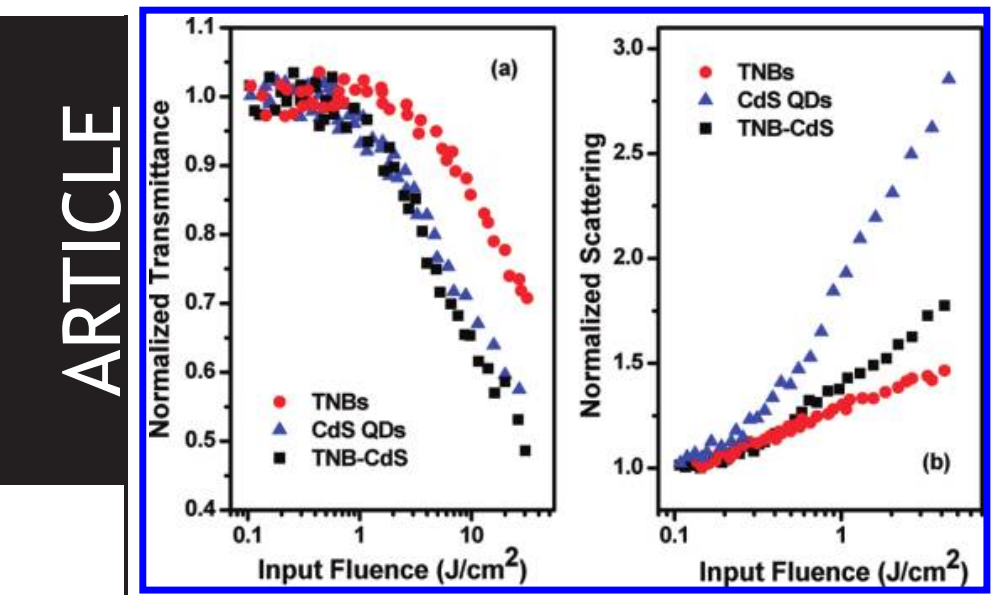

FIGURE 6. (a) Normalized transmittance and (b) nonlinear scattering of TNB, CdS QD, and TNR-CdS samples in aqueous solution at 532 $\mathrm{nm}$. The linear transmittance of each sample was individually adjusted to $70 \%$.

Table 1. Comparison of the NLO Parameters of the TNB, CdS QD, and TNB-CdS Samples in Aqueous Solution at a Wavelength of $532 \mathrm{~nm}$

\begin{tabular}{lccc}
\hline samples & $\begin{array}{c}\text { OL threshold }^{a} \\
\left(\mathrm{~J} / \mathrm{cm}^{2}\right)\end{array}$ & $\begin{array}{c}\text { onset of OL } \\
\left(\mathrm{J} / \mathrm{cm}^{2}\right)\end{array}$ & $\begin{array}{c}T \text { at } \\
5 \mathrm{~J} / \mathrm{cm}^{2}(\%)\end{array}$ \\
\hline TNBs & & 1.45 & 94 \\
CdS QDs & $>31.8$ & 0.56 & 78 \\
TNB-CdS & 31.8 & 0.56 & 74
\end{tabular}

${ }^{a} \mathrm{OL}$ threshold is defined as the input fluence at which the transmittance is $50 \%$ of the linear transmittance.

noted that, on the other side, NLS is strongly dependent on size and plays a dominant role in the case of bigger NPs in solution at higher excitation energy.

The OL effects of the samples, manifested by plotting the normalized transmittance versus the input energy density, were calculated from the open aperture Z-scan measurements, as shown in Figure 6a. All of the samples present a similar trend, that is, the transmittance remains the same at low input fluence and then decreases as the fluence increases. This is a typical feature of OL materials, indicating that all three samples are potential OL candidates. For quantitative comparison, values of representative OL parameters were extracted from the OL curves and listed in Table 1. Clearly, the TNBs display the weakest OL effect, whereas the TNB-CdS exhibits a clear enhancement of the overall OL effect over the CdS QDs, although the onset of OL activity occurs synchronously at $0.56 \mathrm{~J} / \mathrm{cm}^{2}$.

Two main mechanisms are generally accepted to be responsible for the OL effect of materials: NLA and NLS $(15 \mathrm{c})$. For the optical nonlinearities in the semiconductor QDs, NLA, such as FCA, TPA, or MPA, was found to be the major mechanism that is largely affected by the surface states of the QDs (18c, 18d). In contrast, scattering may play the most important role in the OL behavior of nanomaterials. CNTs have been extensively studied as OL materials because of their large NLS performance. To confirm the contribution of NLS to the observed OL activities of the samples, we collected a fraction of the light scattered by the three samples at a forward planar angle of $45^{\circ}$ to the plane of the incident light. Actually, the scattering signals can be observed at different angles from 10 to $180^{\circ}$. The scattering signals with the front scattering directions is much larger than the back scattering directions (39). Generally, with the same scattering angles, the scattering signals are comparable. Figure 6b shows that for all of the samples the intensity of the scattered light increased significantly as the input laser fluences increased. Although nanoscale particles cannot effectively scatter the laser beam according to the Mie scattering theory because of the small size of the scattering centers, the NLS of the CdS QDs clearly outperforms those of the TNBs and TNB-CdS samples. Therefore, it is very interesting that despite the smaller contribution from NLS, the OL effect of TNB-CdS was larger than that of the CdS QDs at the same linear transmittance of $70 \%$ at $532 \mathrm{~nm}$.

As aforementioned, the surface assembly of CdS QDs enhances the fluorescence from the TNBs in the TNB-CdS composite nanostructure. It is conjectured that NLA plays a major role in the optical nonlinearity of TNB-CdS because of efficient charge/energy transfer at the CdS/TNB interface. This is because photoinduced electron transfer could produce a charge-separated excited state to enhance the OL effect. These results are consistent with those of previous studies on a semiconducting polymer/methano-fullerene (40), porphyrin-single walled carbon nanotube (20a), and metal sulfide-multi walled carbon nanotube systems (21b,21c). The origins of NLA vary widely. It may be associated with RSA, FCA, TPA, or MPA. The reason for the OL enhancement caused by photoinduced excitation may be the valence change of the CdS QDs upon excitation contributing to the heterostructure electron system of TNB-CdS. Consequently, when considered as a whole, more than one nonlinear process is responsible for the OL behavior of the TNB-CdS composite nanostructure. Nonlinear refraction is another possible contributor to the OL properties of the TNB-CdS sample.

\section{CONCLUSION}

Facile assembly of CdS QDs on TNBs was achieved by exploiting electrostatic interactions, using a process that can readily be extended to fabricate novel TNM-nanoparticle composite nanostructures with tailored electronic or optical properties. On the basis of data obtained using TEM, XRD, and Raman spectroscopy, uniform decoration of CdS QDs on the TNB surface was achieved. The linear optical properties of the as-prepared TNB-CdS composite were studied by UV-vis absorption and PL spectroscopy. In this composite nanostructure, the luminescence of photoexcited CdS QDs is completely quenched, possibly by a charge/energy transfer process from the QDs to the TNBs, resulting in enhanced blue fluorescence from the TNBs. The NLO properties measured using open aperture z-scan experiments revealed that TNB-CdS possesses an OL effect at $532 \mathrm{~nm}$ that is superior to that of CdS QDs and TNBs alone. This is mainly arises from the combination of NLS and enhanced NLA resulting from efficient charge/energy transfer at the CdS/TNB interface. The unique structure and interesting electro-optical properties of the TNB-CdS composite, render 
such conjugates competitors in the realms of photocatalysis, solar energy conversion, and electrochromic devices as well as for OL applications.

Acknowledgment. This work was supported by the Natural Science Foundation of Fujian Province (2009J01241) and the Fund of National Engineering Research Center for Optoelectronic Crystalline Materials (2005DC105003), the Guangdong Natural Science Foundation (9151007006000009), and Guangdong Provincal Science and Technology (2009B010900047).

\section{REFERENCES AND NOTES}

(1) (a) Law, M.; Greene, L. E.; Johnson, J. C.; Saykally, R.; Yang, P. D Nat. Mater. 2005, 4, 455-459. (b) Bavykin, D. V.; Friedrich, J. M. Walsh, F. C. Adv. Mater. 2006, 18, 2807-2824. (c) Pradhan, S. K.; Mao, Y. B.; Wong, S. S.; Chupas, P.; Petkov, V. Chem. Mater. 2007, $19,6180-6186$.

(2) Liu, S. M.; Gan, L. M.; Liu, L. H.; Zhang, W. D.; Zeng, H. C. Chem. Mater. 2002, 14, 1391-1397.

(3) Shen, Q.; Sato, T.; Hashimoto, M.; Chen, C. C.; Toyoda, T. Thin Solid Films 2006, 499, 299-305.

(4) Kasuga, T.; Hiramatsu, M.; Hoson, A.; Sekino, T.; Niihara, K. Langmuir 1998, 14, 3160-3163.

(5) Kaito, R.; Miyamoto, N.; Kurodaa, K.; Ogawa, M. L. Mater. Chem 2002, 12, 3463-3468.

(6) Miyamoto, N.; Kuroda, K.; Ogawa, M. L. Mater. Chem. 2004, 14, $165-170$.

(7) Kudo, A.; Kaneko, E. Chem. Commun. 1997, 4, 349-350

(8) Nakato, T.; Kusunoki, K.; Yoshizawa, K.; Kuroda, K.; Kaneko, M. L.Phrs. Chem. 1995, 99, 17896-17905.

(9) (a) Xiao, M. W.; Wang, L. S.; Wu, Y. D.; Huang, X. J.; Dang, Z. Nanotechnologv 2008, 19, 015706 -7. (b) Kukovecz, K.; Hodos, M. R.; Konya, Z.; Kiricsi, I. Chem. Phvs. Lett. 2005, 411, 445-449.

(10) Nian, J. N.; Chen, S. A.; Tsai, C. C.; Teng, H. S. L. Phvs. Chem. B 2006, 110, 25817-25824

(11) Ma, R. Z.; Sasaki, T.; Bando, Y. L. Am. Chem. Soc. 2004, 126, 10382-10388.

(12) (a) Byrne, M. T.; McCarthy, J. E.; Bent, M.; Blake, R.; Gun'ko, Y. K.; Horvath, E.; Konya, Z.; Kukovecz, A.; Kiricsi, I.; Coleman, J. N. L. Mater. Chem. 2007, 17, 2351-2358. (b) Shi, Z. O.; Gao, X. P.; Song, D. Y.; Zhou, Y. F.; Yan, D. Y. Polvmer 2007, 48, 7516-7522.

(13) (a) Gao, Y. C.; Wang, Y. X.; Song, Y. L.; Li, Y. L.; Qu, S. L.; Liu H. B.; Dong, B.; Zu, J. F. Opt. Commun. 2003, 223, 103-108. (b) Shirk, J. S.; Lindle, J. R.; Bartoli, F. J.; Kafafi, Z. H.; Snow, A. W. In Materials for Nonlinear Optics-Chemical Perspectives; Marder, S. R., Sohn, J. E., Stucky, G. D., Eds.; ACS Symposium Series; American Chemical Society: Washington, D.C., 1991; Vol. 455, pp626-634. (c) Chen, Y.; EI-Khouly, M. E.; Doyle, J. J.; Lin, Y.; Liu, Y.; Notaras, E.; Blau, W. J.; O’Flaherty, S. M. Phthalocyanines and related compounds: nonlinear optical response and photoinduced electron transfer process. In Handbook of Organic Electronics and Photonics; American Scientific Publishers: Stevenson Ranch, CA, 2008; Vol. 2, pp 151-181. (d) Tutt, L. W.; Boggess, T. F. Prog Ouant. Electron. 1993, 17, 299-338.

(14) (a) Spangler, C. W. I. Mater. Chem. 1999, 9, 2013-2020. (b) Chen, Y.; Hanack, M.; Araki, Y.; Ito, O. Chem. Soc. Rev. 2005, 34, $517-$ 529.

(15) (a) Neto, N. M. B.; Mendonca, C. R.; Misoguti, L.; Zilio, S. C. Appl. Phvs. B: Lasers Opt. 2004, 78, 1-3. (b) Chen, Y.; Lin, Y.; Liu, Y.; Doyle, J.; He, N.; Zhuang, X.; Bai, J.; Blau, W. J. L. Nanosci. Nanotechnol. 2007, 7, 1268-1283. (c) Wang, J.; Chen, Y.; Blau, W. J. L. Mater. Chem. 2009, 19, 7425-7443.

(16) Song, Y. L.; Fang, G. Y.; Wang, Y. X.; Liu, S. T.; Li, C. F.; Song, L. C.; Zhu, Y. H.; Hu, Q. M. Appl. Phvs. Lett. 1999, 74, 332-334

(17) (a) Liu, Z. B.; Wang, Y.; Zhang, X. L.; Xu, Y. F.; Chen, Y.S.; Tian, J. G. Appl. Phvs. Lett. 2009, 94, 021902-1-3. (b) Wang, J.; Hernandez, Y.; Lotya, M.; Coleman, J. N.; Blau, W. I. Adv. Mater. 2009, 21, 2430-2453.

(18) (a) Qu, S. L.; Du, C. M.; Song, Y. L.; Wang, Y. X.; Gao, Y. C.; Liu, S. T.; Li., Y. L.; Zhu, D. B. Chem. Phvs. Lett. 2002, 356, 403-408. (b) Polavarapu, L.; Xu, Q. H.; Dhoni, M. S.; Ji, W. Appl. Phys. Lett.
2008, 92, 263110-1-3. (c) Venkatram, N.; Rao, D. N.; Akundi, M. A. Opt. Express 2005, 13, 867-872. (d) Jia, W. L.; Douglas, E. P.; Guo, F. G.; Sun, W. F. Appl. Phvs. Lett. 2004, 85, 63266328.

(19) Hagan, D. J.; Xia, T.; Said, A. A.; Wei, T. H.; Van Stryland, E. W L. Nonlinear Opt. Phys. Mater. 1993, 2, 483-501.

(20) (a) Liu, Z. B.; Tian, J. G.; Guo, Z.; Ren, D. M.; Du, F.; Zheng, J. Y.; Chen, Y. S. Adv. Mater. 2008, 20, 511-515. (b) Guo, Z.; Du, F. Ren, D. M.; Chen, Y. S.; Zheng, J. Y.; Liu, Z. B.; Tian, J. G. L. Mater. Chem. 2006, 16, 3021-3030.

(21) (a) Chin, K. C.; Gohel, A.; Chen, W. Z.; Elim, H. I.; Ji, W.; Chong, G. L.; Sow, C. H.; Wee, A. T. S. Chem. Phvs. Lett. 2005, 409, 8588. (b) Wu, H. X.; Cao, W. M.; Chen, Q.; Liu, M. M.; Qian, S. X.; Jia, N. Q.; Yang, H.; Yang, S. P. Nanotechnologv 2009, 20, 1956041-10. (c) Feng, M.; Sun, R. Q.; Zhan, H. B.; Chen, Y. Carbon 2010, 48, 1177-1185.

(22) Feng, M.; Sun, R. Q.; Zhan, H. B.; Chen, Y. Nanotechnologv 2010, 21, 075601-1-7.

(23) (a) Skirtach, A. G.; Déjugnat, C.; Braun, D.; Susha, A. S.; Rogach, A. L.; Sukhorukov, G. B. L. Phvs. Chem. C 2007, 111, 555-564. (b) Radziuk, D.; Shchukin, D. G.; Skirtach, A.; Möhwald, H.; Sukhorukov, G. Langmuir 2007, 23, 4612-4617.

(24) Wu, D.; Liu, J.; Zhao, X. N.; Li, A. D.; Chen, Y. F.; Ming, N. B. Chem. Mater. 2006, 18, 547-553.

(25) Colvin, V. L.; Goldstein, A. N.; Alivisatos, A. P. L. Am. Chen. Soc. 1992, 114, 5221-5230

(26) Mao, Y. B.; Wong, S. S. I. Am. Chem. Soc. 2006, 128, 8217-8226.

(27) Zhu, H. Y.; Lan, Y.; Gao, X. P.; Ringer, S. P.; Zheng, Z. F.; Song, D. Y.; Zhao, J. C. I. Am. Chem. Soc. 2005, 127, 6730-6736.

(28) Kolen'ko, Y. V.; Kovnir, K. A.; Gavrilov, A. I.; Garshev, A. V.; Frantti, J.; Lebedev, O. I.; Churagulov, B. R.; Tendeloo, O. G. V.; Yoshimura, M. L. Phvs. Chem. B 2006, 110, 4030-4038.

(29) (a) Das, K.; De, S. K. I. Phvs. Chem. C 2009, 113, 3494-3501. (b) Serpone, N.; Lawless, D.; Khairutdinov, R. L.Phvs. Chem. 1995, $99,16646-16654$

(30) Hwang, S. H.; Moorefield, C. N.; Wang, P. S.; Jeong, K. U.; Cheng, S. Z. D.; Kotta, K. K.; Newkome, G. R. L. Am. Chem. Soc. 2006 , $128,7505-7509$

(31) Ou, Y. Y.; Huang, M. H. L. Phvs. Chem. B 2006, 110, 2031-2036.

(32) Lee, J. C.; Kim, T. G.; Lee, W.; Han, S. H.; Sung, Y. M. Cryst. Growth. Des. 2009, 9, 4519-4523.

(33) (a) Premachandran, R.; Banerjee, S.; John, V. T.; McPherson, G. L. Chem. Mater. 1997, 9, 1342-1347. (b) Noglik, H.; Pietro, W. L Chem. Mater. 1994, 6, 1593-1595.

(34) Olek, M.; Büsgen, T.; Hilgendorff, M.; Giersig, M. L. Phys. Chem. B 2006, 110,12901-12904

(35) (a) Sudhagar, P.; Jung, J. H.; Park, S.; Lee, Y. G.; Sathyamoorthy, R.; Kang, Y. S.; Ahn, H. Electrochem. Commun. 2009, 11, 2220 2224. (b) Baker, D. R.; Kamat, P. V. Adv. Funct. Mater. 2009, 19 , 805-811

(36) Sheik-Bahae, M.; Said, A. A.; Wei, T. H.; Hagan, D. J.; Van Stryland, E. W. IEEE I. Ouantum Electron. 1990, 26, 760-769.

(37) (a) Liu, Z. B.; Xu, Y. F.; Zhang, X. Y.; Zhang, X.L.; Chen, Y. S. Tian, J. G. L.Phys. Chem. B 2009, 113, 9681-9686. (b) Xu, Y. F. Liu, Z. B.; Zhang, X. L.; Wang, Y.; Tian, J. G.; Huang, Y.; Ma, Y. F.; Zhang, X. Y.; Chen, Y. S. Adv. Mater. 2009, 21, 1275-1279. (c) Senge, M. O.; Fazekas, M.; Notaras, E. G. A.; Blau, W. J.; Zawadzka, M.; Locos, O. B.; Mhuircheartaigh, E. M. N. Adv. Mater. 2007, 19, 2737-2774. (d) Wu, W.; Zhang, S.; Li, Y.; Li, J. X.; Liu, L. Q.; Qin, Y. J.; Guo, Z. X.; Dai, L. M.; Ye, C.; Zhu, D. B Macromolecules 2003, 36, 6286-6288. (e) He, N.; Chen, Y.; Bai, J. R.; Wang, J.; Blau, W. J.; Zhu, J. H. L. Phvs. Chem. C 2009, 113 , $13029-13035$

(38) Jing, C. B.; Xu, X. G.; Zhang, X. L.; Liu, Z. B.; Chu, J. H. I. Phys. D: Appl. Phvs. 2009, 42, 075402-1-6. (b) Neo, M. S.; Venkatram, N.; Li, G. S.; Chin, W. S.; Ji, W. L.Phvs. Chem. C 2009, 113, 1905519060.

(39) (a) Polavarapu, L.; Venkatram, N.; Ji, W.; Xu, Q. H. ACS Appl. Mater. Interfaces 2009, 1, 2298-2303. (b) Singh, C. P.; Bindra, K. S.; Bhalerao, G. M.; Oak, S. M. Opt. Express 2008, 16, 8440 8450 .

(40) Sariciftci, N. S.; Smilowitz, L.; Heeger, A. J.; Wudl, F. Science 1992, 258, $1474-1476$.

AM100003P

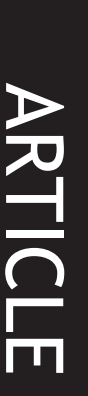

